\title{
Corner point singularities under in-plane and out-of-plane loading: a review of recent results
}

\author{
F. Berto ${ }^{a^{*}}$, Les P. Pook ${ }^{b}$ and A. Campagnolo ${ }^{c}$
}

${ }^{a}$ Department of Mechanical Engineering, NTNU, Trondehim, Norway

${ }^{b} 21$ Woodside Road, Sevenoaks TN13 3HF, United Kingdom

${ }^{c}$ Department of Industrial Engineering, University of Padova, Via Venezia 1, 35131, Padova, Italy

\begin{tabular}{l}
\hline A R T I C L EI N F O \\
\hline Article history: \\
Received 6 March, 2017 \\
Accepted 12 June 2017 \\
Available online \\
12 June 2017 \\
\hline Keywords: \\
Finite elements \\
Mixed modes \\
Coupled modes \\
Stress intensity factors \\
Corner point singularities
\end{tabular}

\section{A B S T R A C T}

\begin{abstract}
The linear elastic analysis of homogeneous, isotropic cracked bodies started in the 1900s. The existence of three dimensional corner point effects in the vicinity of a corner point where a crack front intersects a free surface was investigated in the late 1970s. An approximate solution by Bažant and Estenssoro explained some features of corner point effects but there were various paradoxes and inconsistencies. Results derived from finite element models showed that the analysis is incomplete. The stress field in the vicinity of a corner point appears to be the sum of two different singularities (i.e. stress intensity factors and corner point singularities). In this paper some recent results for the corner point singularities under in and out of plane loadings is reviewed and discussed.
\end{abstract}

\section{Introduction}

The linear elastic analysis of homogeneous, isotropic cracked bodies is a Twentieth Century development (Pook, 2013, 2015), with the first papers appearing in 1907, but it was not until the introduction of the stress intensity factor concept in 1957 (Williams, 1957) that widespread application of linear elastic fracture mechanics (LEFM) to practical engineering problems became possible. A stress intensity factor is the leading term of a series expansion of a crack tip stress field. The first application of finite elements to the calculation of stress intensity factors for two dimensional cases was in 1969 (Dixon \& Pook, 1969). Finite element analysis had a significant influence on the development of LEFM. Corner point singularities were investigated in the late 1970s (Bažant \& Estenssoro, 1979). It was soon found that the existence of corner point effects made interpretation of calculated stress intensity factors calculated using finite element analysis difficult, and their validity questionable.

* Corresponding author.

E-mail addresses: filippo.berto@ntnu.no (F. Berto) 
One of the main interests in fracture mechanics is to quantify the crack tip surface displacement (Pook, 2000). By superimposing the displacements due to the three modes of loading (mode I, mode II, mode III), shown in Fig. 1, it is possible to fully describe the crack tip surface displacements. If a crack surface is considered as consisting of points then the three modes of crack surface displacement provide an adequate description of the movements of crack surfaces when a load is applied. Assuming a Poisson's ratio, $v$, greater than zero, and also assuming that the crack front is perpendicular to a surface of a body, as is done in this paper, then it is possible to prove that modes II and III at the crack tip cannot exist in isolation (Kotousov et al., 2013; Lazzarin \& Zappalorto, 2012). Mode II causes mode III $^{\mathrm{c}}$ and mode III generates mode II $^{\mathrm{c}}$. These induced modes are properly named coupled modes. The superscript $\mathrm{c}$ is usually employed for their representation. There are no coupled modes when $v$ is zero, and the magnitude of coupled modes increases with $v$ (Kotousov et al., 2012). It is not clear what happens when $v$ is less than zero.

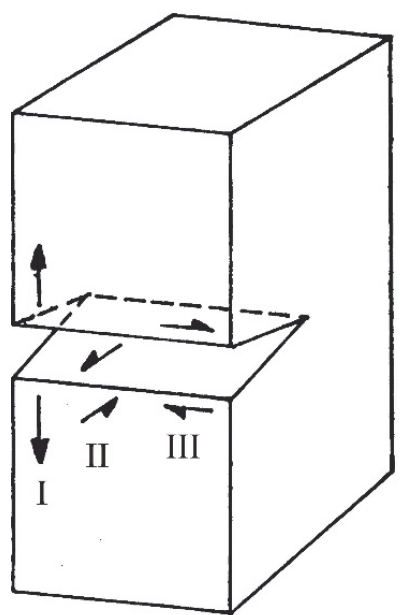

Fig. 1. Notation for modes of crack tip surface displacement.

A series expansion is able to represent the stress field in the neighborhood of the crack tip (Williams, 1957; Paris \& Sih, 1965). The leading order term of this series is the stress intensity factor, $K$. Subscripts I, II, II are used to denote mode. In agreement with the well known fracture mechanics framework, the stress components are proportional to $K / \sqrt{r}$ where $r$ is the distance from the crack tip (Fig. 2). Displacements are instead proportional to $K \sqrt{r}$. The leading order term tied to the stress intensity factor provides an accurate description of the stress field in a $K$-dominated region characterized by a radius $r \approx a / 10$ where $a$ is crack length, (Pook, 2000).

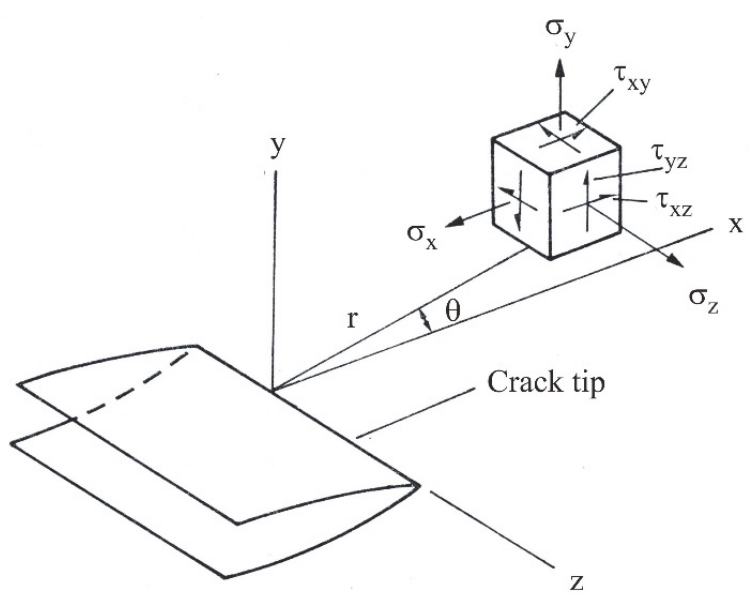

Fig. 2. Notation for crack tip stress field 
The concept of a corner point singularity was introduced by Bažant and Estenssoro (1979) first and later by Pook (1994). Some pioneering results were given also by Benthem (1980). An approximate solution showed that the intensity of the stress field near the point of corner singularity can be done by defining a stress intensity measure called $K_{\lambda}$. However, explicit expressions for $K_{\lambda}$, and the stress and displacement fields associated to it are not available. The only statement based on the initial assumption used is that stresses are proportional to $K_{\lambda} r^{\lambda}$ and displacements to $K_{\lambda} r^{1-\lambda}$. The distance $r$ is in this particular case the distance of a generic point from the corner point and $\lambda$ is a function of Poisson's ratio.

For a crack surface intersection angle, $\gamma$ of $90^{\circ}$ and a crack front intersection angle $\beta$ of $90^{\circ}$, defined as in Figs. (3-4) there are two modes of stress intensity measure. These are the symmetric mode, stress intensity measure $K_{\lambda \mathrm{S}}$, where crack tip surface displacements are mode 1 (Fig. 1), and the antisymmetric mode, stress intensity measure $K_{\lambda \mathrm{A}}$, which is a combination of modes II and III crack tip surface displacements. For the special case of $\lambda=0.5$, stress intensity factors are recovered. $K_{\lambda \mathrm{S}}$ becomes $K_{\mathrm{I}}$, and $K_{\lambda \mathrm{A}}$ a combination of $K_{\mathrm{II}}$ and $K_{\text {III. }}$. In the remainder of the paper $\gamma$ and $\beta$ are both $90^{\circ}$ unless otherwise stated.

Application of Bažant and Estenssoro's analysis was successful in explaining some aspects of behavior in the vicinity of a corner point (Pook, 2013). For example, the parameter $\lambda$ is a function of Poisson's ratio, $v$, and the crack front intersection angle, $\beta$ (Fig. 4). At a critical crack front intersection angle, $\beta_{\mathrm{c}}, \lambda=0.5$ and stress intensity factors then have finite values in the corner point region (Pook, $2011)$. For $\beta<\beta_{\mathrm{c}}, \lambda<0.5$ so $K_{\mathrm{I}}$ tends to zero as a corner point is approached. Hence for a propagating crack, crack propagation away from the corner point is favoured. For $\beta>\beta_{\mathrm{c}}, \lambda>0.5 K_{\mathrm{I}}$ tends to infinity as a corner point is approached and crack propagation at the corner point is favoured. In consequence, for a propagating crack the crack front shape adjusts itself such that the crack front intersection angle tends to $\beta_{\mathrm{c}}$. This accounts for the bowed crack fronts observed in fatigue precracks in fracture toughness test pieces. An example is shown in Fig. 5 (Pook, 1968).
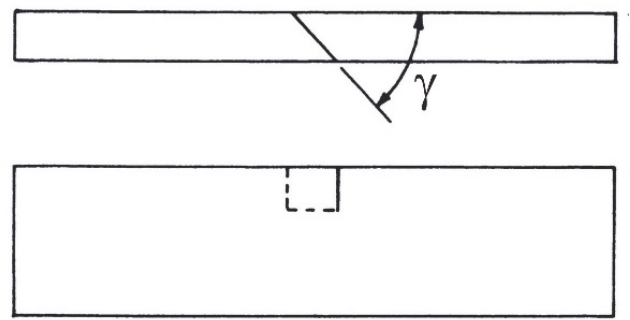

Fig. 3. Angle crack test piece, definition of crack Fig. 4. Definition of crack front intersection surface intersection angle, $\gamma$ angle, $\beta$
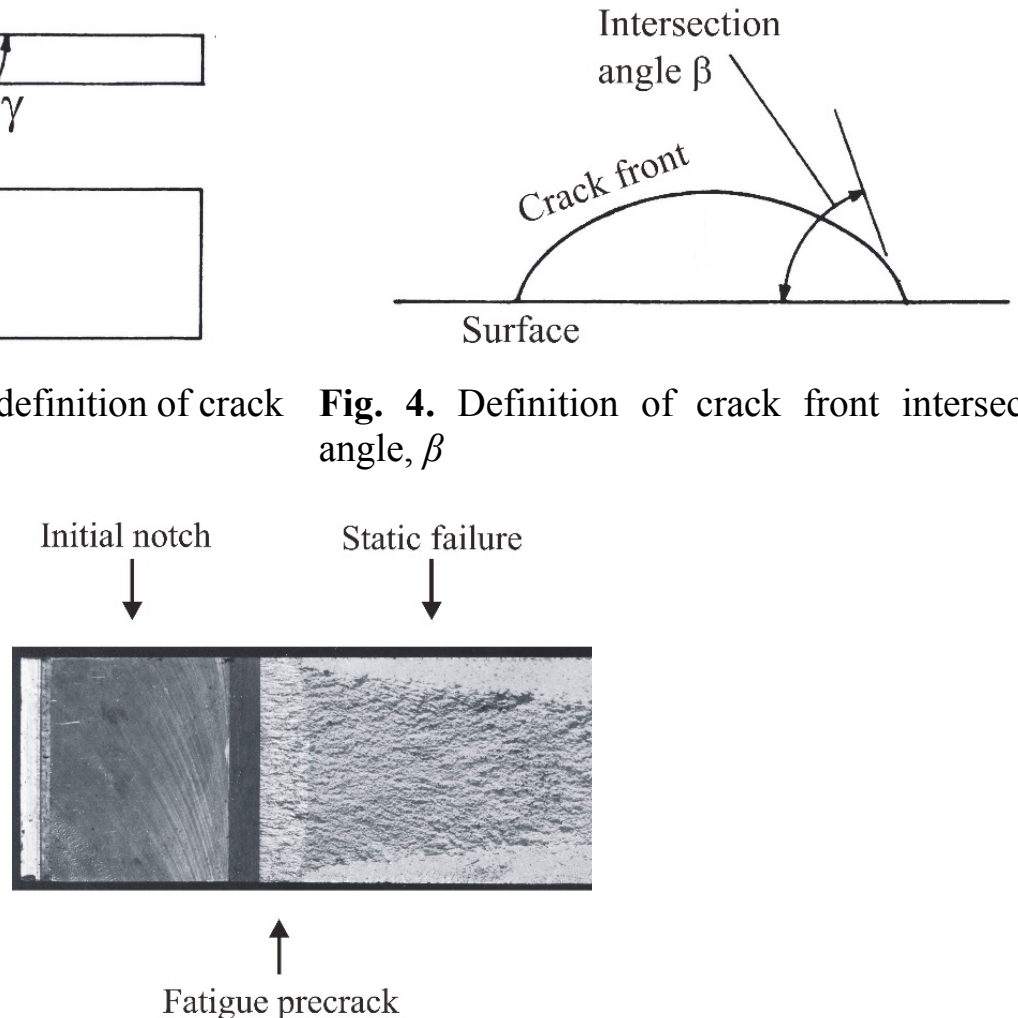

Fatigue precrack

Fig. 5. Fracture surface of $19 \mathrm{~mm}$ thick aluminium alloy fracture toughness test piece 
Bažant and Estenssoro's analysis was not completely successful in that various paradoxes and inconsistencies appeared (Pook, 2013). For example, their analysis predicts that for a crack front intersection angle of $90^{\circ}$ (Fig. 4) $K_{\text {III }}$ theoretically tend to infinity as corner point is approached. This is apparently confirmed by the finite element results shown in Fig. 6 (Pook, 2013). This is paradoxical because shear stresses must be zero perpendicular to a free surface so $K_{\text {III }}$ should also be zero.

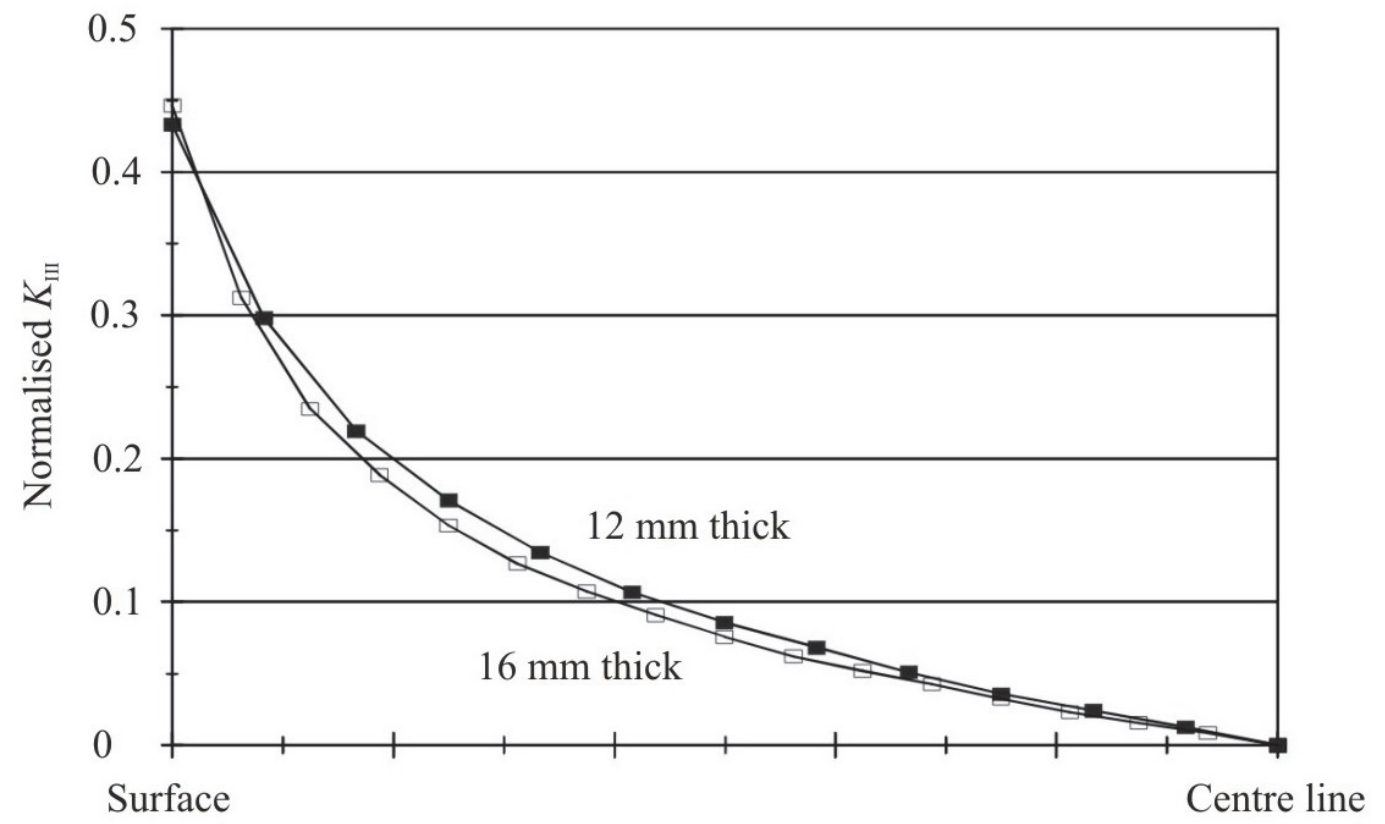

Fig. 6. Through thickness distribution of induced $K_{\text {III }}$ for $45 \mathrm{~mm}$ wide, $12 \mathrm{~mm}$ and $16 \mathrm{~mm}$ thick, crack length $20 \mathrm{~mm}$ models, mode II loading

In general, it is quite usual for the results of finite element analyses to show abrupt changes of stress components in the vicinity of a corner point where a crack or notch intersects a free surface. For example, Fig. 7 shows some results for finite element analyses on a notched disc $200 \mathrm{~mm}$ diameter and $50 \mathrm{~mm}$ thick containing a narrow radial notch $100 \mathrm{~mm}$ long. Displacements corresponding to mode II displacements for $K_{\mathrm{II}}=1000 \mathrm{MPa} \sqrt{\mathrm{mm}}$ in an infinite body were applied to the nodes on the edge of the disc. Calculations were carried out for notch tip radii of $1 \mathrm{~mm}, 0.5 \mathrm{~mm}$ and $0.1 \mathrm{~mm}$ and, for comparison, on a cracked disc. Results for the induced through thickness shear stress, $\tau_{\mathrm{yz}}$, at $0.3 \mathrm{~mm}$ from the notch or crack tip in the $x$ direction, are shown in Fig. 7. All four data sets are similar. There are clearly defined maxima at between $1.8 \mathrm{~mm}$ and $2.7 \mathrm{~mm}$ from the surface. The results give an indication of the through thickness distribution of induced $K_{\text {III. }}$. Values increase from zero at the center line, where $K_{\text {III }}$ changes sign, until they reach a maximum near the surface.

In an attempt to resolve paradoxes and inconsistencies an extensive finite element research program has been carried out (Pook et al., 2014, 2015, 2016). The purpose of the present paper is to present some of the results obtained, and also to present a possible approach to resolution of paradoxes and inconsistencies.

\section{The research program}

Two finite element models were considered. One quarter of each was modelled (Fig. 8). Firstly, discs of finite thickness under anti-plane (remote nominal mode III) loading (Pook et al., 2014). The radius of the disc, $r$, is equal to $50 \mathrm{~mm}$. A through the thickness crack with its tip at the center of the disc has been considered, with a length, $a$, equal to $50 \mathrm{~mm}$. Different ratios have been considered between the disc thickness $t$ and the crack length $a$. In particular the following ratios have been modelled: $t / a=0.25,0.5,0.75,1,1.25,1.5,1.75,2,2.25,2.5,2.75$ and 3 . Finite element models have 
been analysed by means of ANSYS 11. Stress intensity factors were evaluated from the stress components on the crack surface, in the neighbourhood of the crack tip, using standard equations (Pook, 2000; Paris \& Sih 1965). The material has been considered linear elastic and the Poisson's ratio has been set equal to 0.3 while the Young's modulus, $E$, has been set equal to $200 \mathrm{GPa}$. The load has been applied in terms of displacement on the nodes corresponding to the cylindrical surfaces. The applied displacements correspond to a nominal mode III stress intensity factor $\mathrm{K}_{\mathrm{III}}=1 \mathrm{MPa} \cdot \mathrm{m}^{0.5}(31.62$ $\mathrm{N} \cdot \mathrm{mm}^{0.5}$ ). Recently, results have been obtained for in-plane shear loading of a disc, $\mathrm{t} / \mathrm{a}=1$ (Pook et al. 2016). In plane displacements were applied on the cylindrical surface, corresponding to a nominal mode II stress intensity factor $\mathrm{K}_{\mathrm{II}}=1 \mathrm{MPa} \cdot \mathrm{m}^{0.5}\left(31.62 \mathrm{~N} \cdot \mathrm{mm}^{0.5}\right)$.

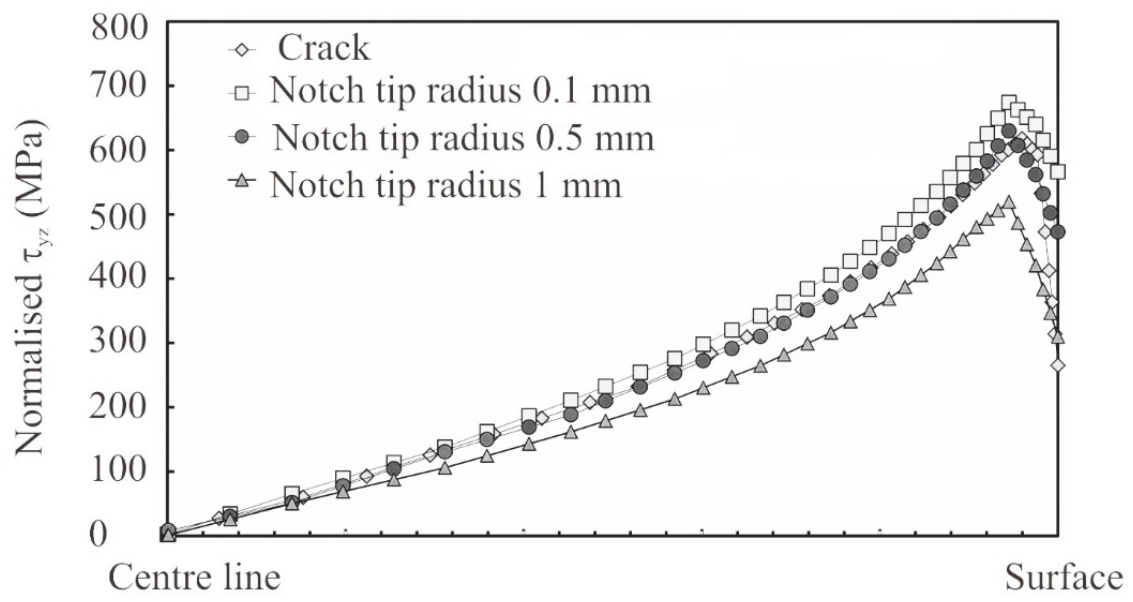

Fig. 7. Distribution of the through thickness shear stress, $\tau_{\mathrm{yz}}, 0.3 \mathrm{~mm}$ from the notch or crack tip for notched and cracked plates, mode II loading
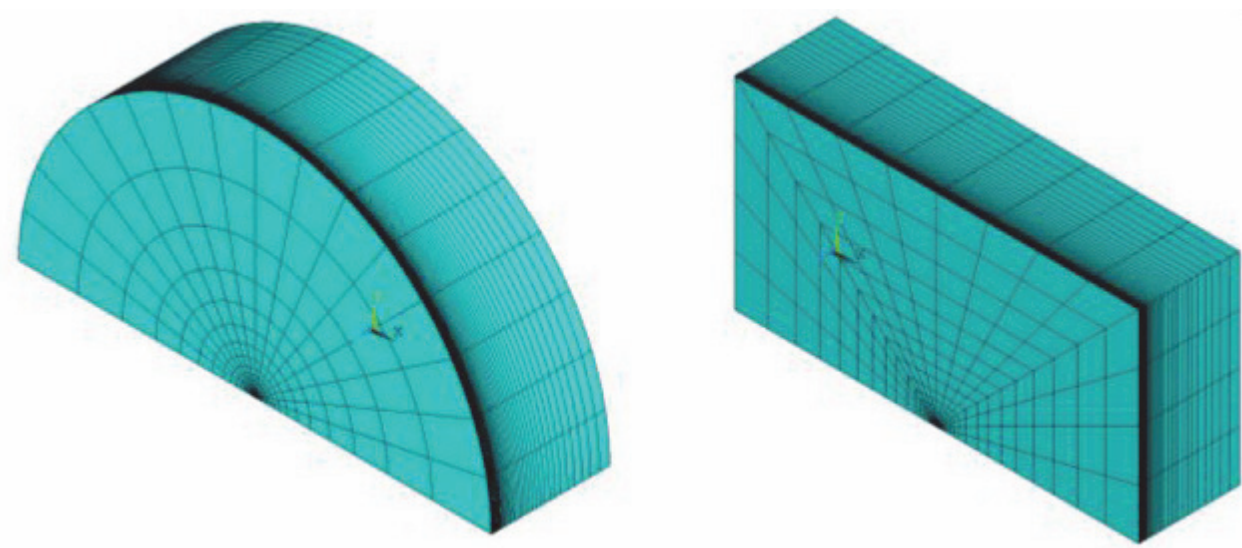

Fig. 8. Finite element models. Left, disc, Right, plate

Secondly, plates of finite thickness under anti-plane (remote nominal mode III) (Pook et al., 2015). A square plate with a constant width equal to $100 \mathrm{~mm}$ has been considered. The thickness of the plate $t$ has been varied in the models keeping constant the crack length $(a=50 \mathrm{~mm})$. The following ratios between $t$ and $a$ have been considered: $0.25,0.5,0.75,1,1.25,1.5,1.75,2,2.25,2.5,2.75$ and 3 . As for the case of the discs the material has been considered obeying a linear elastic law with $v=0.3$ and $E=200 \mathrm{GPa}$. In this case the load has been applied by means of a constant displacement equal to $10^{-3}$ $\mathrm{mm}$ applied on the external edge of the plate. Displacements $z$ were applied to the side containing the crack mouth.

Stress components, $\tau_{\mathrm{yz}}$ and $\tau_{\mathrm{xy}}$ were obtained from finite element models at different distances, $s$, from the free surfaces of the plate or disc. In particular, distances of $0 \mathrm{~mm}, 0.25 \mathrm{~mm}, 1 \mathrm{~mm}$ and $2 \mathrm{~mm}$ have were considered. Typical results are shown in Figure 9, plotted on logarithmic scales. The smallest 
172

distance from the crack tip is $0.0006 \mathrm{~mm}$. Results from discs and plates are very similar. Values of $\lambda$, the coefficient defining stress intensity measure which were obtained are listed in Table 1, comparing the results from discs and plates. For $s=0$ and $0.25 \mathrm{~mm} \tau_{\mathrm{yz}}$ does not behave as a straight line. For values of s ranging between 0.25 to $2 \mathrm{~mm}$ the slope characterizing $\tau_{\mathrm{xy}}$ is constant and almost equal to the theoretical value of 0.5 . Hence, Mode II stress intensity factor $K_{\text {II }}$ can be correctly defined and calculated for these values of $s$. Dealing with cracked plates at $s=0$ the slope $\lambda$ reaches its maximum at $t / a=0.25$ remaining in all the cases considered significantly lower than the value of 0.598 usually assumed for the case of a corner point singularity. These latest results are different from those obtained in the case of discs where the slope $\lambda$ increases for increasing values of $t / a$. Both for plates and discs the mode III stress intensity factor $K_{\text {III }}$ can be well defined at $s=1 \mathrm{~mm}$ and $2 \mathrm{~mm}$ because the slope is close to that theoretically expected (0.5). For in plane shear loading stresses $\tau_{\mathrm{yz}}$ and $\tau_{\mathrm{xy}}$ on the crack surface at $s=0 \mathrm{~mm}$ are similar to the stress distributions for nominal mode III loading. The value of $\lambda$, calculated from $\tau_{\mathrm{xy}}$, is 0.541 , is virtually the same as for nominal mode III loading.

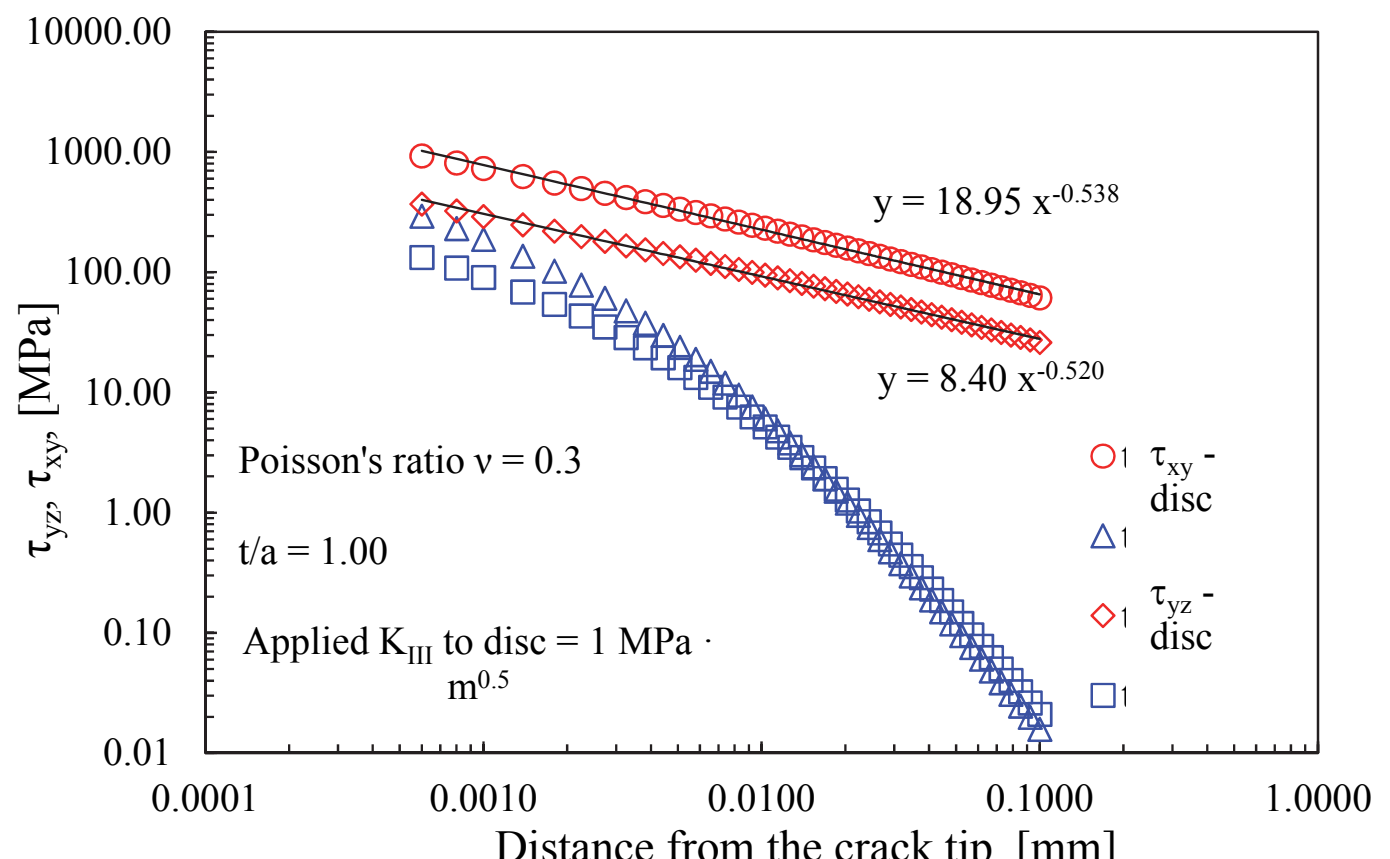

Fig. 9. Stresses $\tau_{\mathrm{yz}}$ and $\tau_{\mathrm{xy}}$ on crack surface at $s=0 \mathrm{~mm}$ from disc and plate surfaces, $t / a=1$

Table 1. Values of $\lambda$ for $\tau_{\mathrm{xy}}, s$ is the distance from the surface in the $\mathrm{z}$ direction. Values in brackets are for $\tau_{\mathrm{yz}}$

\begin{tabular}{lcccccc}
\hline \multirow{2}{*}{$t / a$} & \multicolumn{2}{c}{$s=0 \mathrm{~mm}$} & \multicolumn{2}{c}{$s=0.25 \mathrm{~mm}$} & \multicolumn{2}{c}{$s=1 \mathrm{~mm}$} \\
\hline & disc & plate & disc & plate & disc & plate \\
\hline 0.25 & 0.505 & 0.538 & 0.497 & 0.498 & $0.497(0.508)$ & $0.497(0.507)$ \\
0.50 & 0.520 & 0.527 & 0.497 & 0.498 & $0.497(0.506)$ & $0.497(0.506)$ \\
0.75 & 0.530 & 0.523 & 0.497 & 0.498 & $0.497(0.507)$ & $0.497(0.506)$ \\
1.00 & 0.538 & 0.520 & 0.497 & 0.498 & $0.497(0.507)$ & $0.497(0.506)$ \\
1.25 & 0.544 & 0.517 & 0.497 & 0.498 & $0.497(0.506)$ & $0.497(0.506)$ \\
1.50 & 0.549 & 0.515 & 0.497 & 0.498 & $0.497(0.506)$ & $0.497(0.506)$ \\
1.75 & 0.553 & 0.513 & 0.497 & 0.498 & $0.497(0.506)$ & $0.497(0.506)$ \\
2.00 & 0.556 & 0.512 & 0.497 & 0.498 & $0.497(0.506)$ & $0.497(0.506)$ \\
2.25 & 0.559 & 0.510 & 0.497 & 0.498 & $0.497(0.507)$ & $0.497(0.506)$ \\
2.50 & 0.542 & 0.510 & 0.497 & 0.498 & $0.497(0.506)$ & $0.497(0.505)$ \\
2.75 & 0.545 & 0.509 & 0.497 & 0.498 & $0.497(0.506)$ & $0.497(0.506)$ \\
3.00 & 0.547 & 0.508 & 0.497 & 0.498 & $0.497(0.507)$ & $0.497(0.506)$ \\
\hline
\end{tabular}




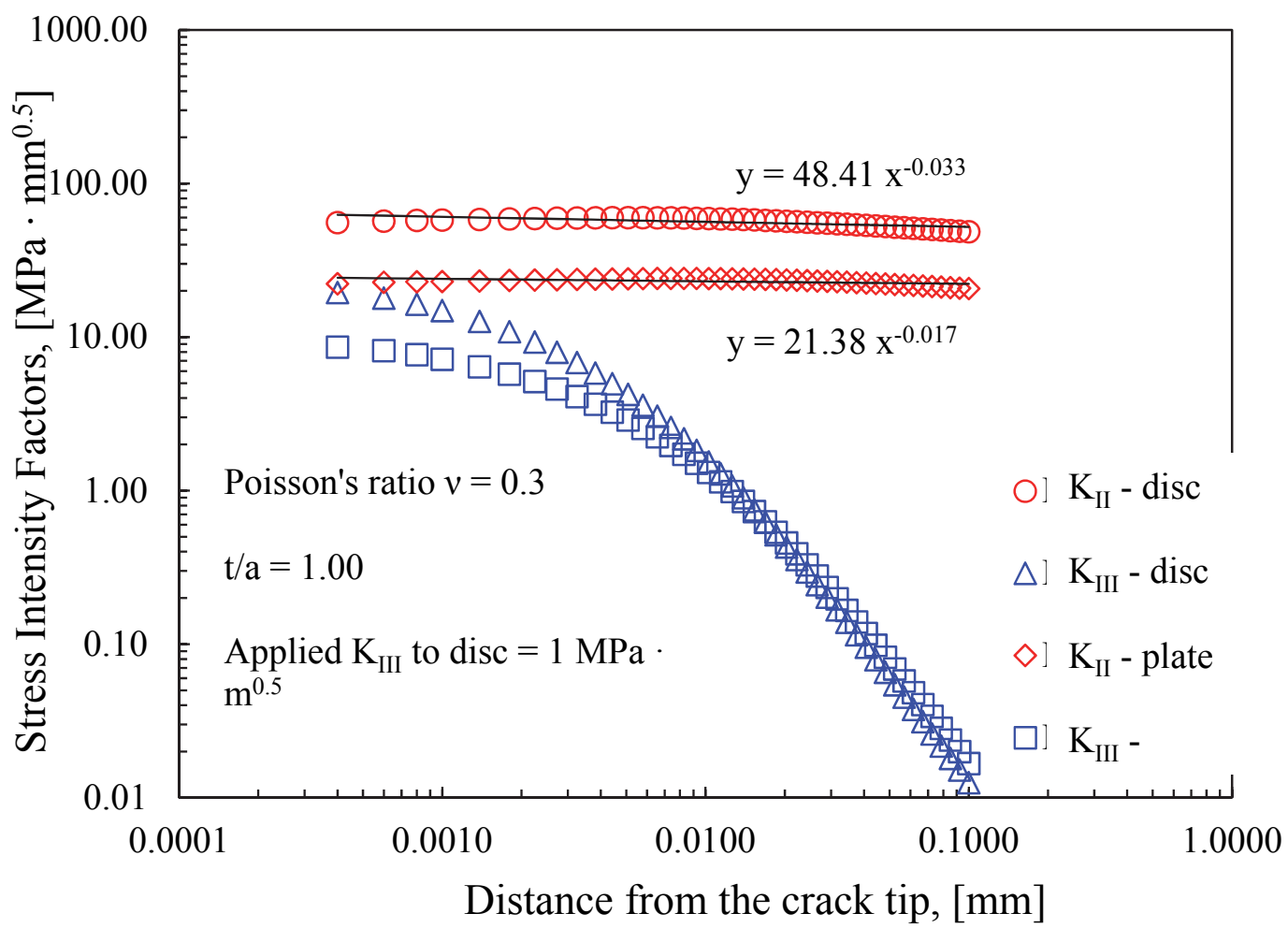

Fig. 10. $K_{\mathrm{II}}$ and $K_{\mathrm{III}}$ at $s=0 \mathrm{~mm}$ from disc and plate surfaces, $t / a=1$

The finite element results obtained at the coordinate $s=0$ (Fig. 9) show that the stress component $\tau_{\mathrm{yz}}$ is very far from the linear theoretical trend corresponding to a straight line on log coordinates. This is true both for discs and plates. The corresponding stress intensity factors are shown in Fig. 10. The apparent value of the mode III stress intensity factor $K_{\text {III }}$ is strongly dependent on the distance from the crack tip for which it is obtained. For $s=0$ realistic values of $K_{\text {III }}$ cannot be calculated. Values of $\lambda$, calculated from $\tau_{\mathrm{xy}}$ and $\tau_{\mathrm{yz}}$, are 0.499 and 0.506 , in excellent agreement with the nominal mode III results. Similarly, stresses $\tau_{\mathrm{yz}}$ and $\tau_{\mathrm{xy}}$ on the crack surface at $s=2 \mathrm{~mm}$ from the disc are similar to the stress distributions for nominal mode III loading. Values of $\lambda$ are in excellent agreement. Distributions of $K_{\mathrm{II}}$ and $K_{\mathrm{III}}$ at $s=0 \mathrm{~mm}$ from the disc surface are similar to those for nominal mode III loading, shown in Fig. 10. However, through the thickness distribution of $K_{\text {II }}$ and $K_{\text {III }}$, differ from those for nominal mode III loading (Pook et al. 2014,2015). This difference is because the use of nominal mode II loading has eliminated disc bending. The results show that the change of loading mode from nominal mode III to nominal mode II has had no effect on the distributions of $\tau_{\mathrm{yz}}$ and $\tau_{\mathrm{xy}}$ on and near the crack surface, but has significantly changed the through thickness distributions of $K_{\text {II }}$, $K_{\text {III. Similar }}$ observations have been also reported for other mixed mode test geometries like edge notched disc bend (ENDB) specimen and tested with different brittle materials (Aliha et al., 2015a,b, 2016a,b, 2017; Bahmani et al., 2017).

\section{Discussion}

At the present state of the art the results obtained show that Bažant and Estenssoro's solution for corner point singularities is incomplete (Pook et al. 2014,2015, 2016). For mode I loading the analysis successfully predicts the critical angle at which a crack front intersects a free surface. For simplicity, two dimensional analyses are often use for sheets and plates of constant thickness. However, the bowed crack fronts which are a consequence of corner point singularities (Fig. 5) mean that these are inevitably approximations. Two dimensional analyses are used in fracture toughness test standards (Pook 2013) and this has two consequences. Firstly, very detailed requirements are need to ensure that consistent results are obtained and, secondly, transferability of data to practical situations is limited. Indeed, in 
general, the fact that a particular data set has been successfully analyzed using a two dimensional analysis is no guarantee that the data can be extrapolated to a different situation.

Bažant and Estenssoro's analysis suggests that $K_{\mathrm{III}}$ tends to infinity as a corner point is approached but the results show that this does not happen. In addition it is noticeable that apparent values of $K_{\text {III }}$ in the vicinity of a surface are a strong function of the distance from the crack tip at which they are calculated. It is clear that some of the inconsistencies which have been observed (Pook, 2013) are artefacts of finite element analyses. Stresses at finite element nodes are extrapolations from Gauss points within the element (Anon, 1986). They depend both on the method of extrapolation and on the element size. Inaccuracies in extrapolated stress are a particular problem in regions of high stress gradient. The results, using a fine mesh, shown in Fig. 7 have captured a sharp peak in $\tau_{\mathrm{yz}}, 0.3 \mathrm{~mm}$ from the crack tip. As expected from elementary considerations, $\tau_{\mathrm{yz}}$ appears to tend to zero as the surface is approached. In contrast the values of $K_{\text {III }}$ shown in Fig. 6 , which were calculated from $\tau_{\mathrm{yz}}$ do not show a peak and $K_{\text {III }}$ apparently tends to infinity as the surface is approached, This is because a coarse mesh was used and extrapolation has resulted in a misleading impression that $K_{\text {III }}$ tends to infinity as the surface is approached, which is incorrectly predicted by Bažant and Estenssoro's analyis.

In 1965 it was pointed out that mode III is a torsion problem (Paris \& Sih, 1965). Under mode III loading initially plane cross sections, including the surface at a corner point, do not remain plane under load. It is well known that serious error can arise if warping of non circular cross sections under torsion is not taken into account in stress analyses (Timoshenko \& Goodier, 1970). Warping of the surface under mode III means that $\tau_{\mathrm{yz}}$ at the surface does not have to be zero. This implies that $K_{\mathrm{III}}$ does not have to be zero, but the effect may be too small to be significant. However, Bažant and Estenssoro's theory does need to be refined.

There is no clear pattern to the values of $\lambda$ shown in Table 1 , and attempts to find a three dimensional stress function for the stresses in the vicinity of a corner point have so far been unsuccessful. A corner point stress function could be expected to reduce to a Westergaard stress function as a special case, but the following argument suggests that this is impossible. The Westergaard method of stress analysis (Paris and Sih 1965) makes use of complex variables in which it is assumed that $i^{2}=-1$. Complex numbers can be represented by a point on a plane. A three dimension equivalent to a Westergaard stress function would have to be based on a hypercomplex number system.

Hamilton's quaternions are a hyper complex number system (Pulver, 2008) that is sometimes used in elasticity (Weisz-Patrault et al., 2014). In a quaternion it is assumed that $i^{2}=j^{2}=k^{2}=-1$. A quaternion can be represented by a point in 4 dimensional space. Quaternions can be represented as pairs of complex numbers, but do not include complex numbers as a special case. A three dimensional equivalent of the Westergaard method would have to be based on quaternions. This suggests that a corner point stress function could not include Westergaard stress function as a special case. This implies that stresses in the vicinity of a corner point are sums of stresses due to two different singularities of different orders: stress intensity factors and corner point singularities. The latter being asymptotic to zero as distance from the corner point increases. In other words Bažant and Estenssoro's analysis is incomplete. It is therefore not surprising that values of $\lambda$ derived from finite element analysis are inconsistent. To make progress the next step is to use the finite element results to separate the two singularities numerically and see whether this gives clues to the form of corner point singularities.

Despite substantial theoretical advances during the last five decades, fatigue crack paths in structures, and fatigue crack propagation data, are still often obtained experimentally in a wide range of industries (Pook, 2011). Experimental validation of theoretical crack path and associated crack propagation data through appropriate structural fatigue testing is sometimes a requirement of regulatory authorities. An important reason for this is that corner point singularities are only partly understood. In the absence of a theoretical breakthrough the situation is unlikely to improve significantly in the foreseeable future. 


\section{Conclusion}

(1) At the present state of the art corner point singularities are only partly understood. Results obtained using highly accurate finite element analysis show that Bažant and Estenssoro's solution is incomplete.

(2) Some of the inconsistencies which have been observed are artefacts of finite element analyses due to the use of meshes which are too coarse.

(2) Under mode I loading Bažant and Estenssoro's solution correctly predicts the angle at which a crack front intersects a free surface. This leads to bowed crack fronts in sheets and plates of constant thickness.

(3) Bowed crack fronts in sheets and plates of constant thickness mean that two dimensional analyses are inherently approximations. This limits the transferability of data.

(4) There is no clear pattern to the values of the coefficient $\lambda$, which characterises corner point singularities in Bažant and Estenssoro's solution. Attempts to find a three dimensional stress function which rationalises these values have so far been unsuccessful.

(5) A corner point stress function could be expected to reduce to a Westergaard stress function as a special case. However, this appears to be impossible because Hamilton's quaternions do not reduce to complex numbers as a special case.

(6) Stresses in the vicinity of a corner point appear to be sums of stresses due to two different singularities of different orders: stress intensity factors and corner point singularities. To make progress the next step is to use the highly accurate finite element results to separate the two singularities numerically and see whether this gives clues to the form of corner point singularities.

(7) In the absence of a theoretical breakthrough the situation is unlikely to improve significantly in the foreseeable future.

\section{References}

Aliha, M. R. M., Bahmani, A., \& Akhondi, S. (2015a). Numerical analysis of a new mixed mode I/III fracture test specimen. Engineering Fracture Mechanics, 134, 95-110.

Aliha, M. R. M., Bahmani, A., \& Akhondi, S. (2015b). Determination of mode III fracture toughness for different materials using a new designed test configuration. Materials \& Design, 86, 863-871.

Aliha, M. R. M., Bahmani, A., \& Akhondi, S. (2016a). A novel test specimen for investigating the mixed mode I+ III fracture toughness of hot mix asphalt composites-Experimental and theoretical study. International Journal of Solids and Structures, 90, 167-177.

Aliha, M. R. M., Berto, F., Bahmani, A., Akhondi, S., \& Barnoush, A. (2016b). Fracture assessment of polymethyl methacrylate using sharp notched disc bend specimens under mixed mode I+ III loading. Physical Mesomechanics, 19(4), 355-364.

Aliha, M. R. M., \& Bahmani, (2017). A rock fracture toughness study under mixed mode I/III loading. Rock Mechanics and Rock Engineering, 1-13.

Anon, A. (1986). A finite element primer. East Kilbride, Glasgow: National Engineering Laboratory; 1986.

Bahmani, A., Aliha, M. R. M., \& Berto, F. (2017). Investigation of fracture toughness for a polycrystalline graphite under combined tensile-tear deformation. Theoretical and Applied Fracture Mechanics. Doi: 10.1016/j.tafmec.2017.02.011

Bažant, Z. P., \& Estenssoro, L. F. (1979). Surface singularity and crack propagation. International Journal of Solids and Structures, 15(5), 405-426.

Benthem, J. P. (1980). The quarter-infinite crack in a half space; alternative and additional solutions. International Journal of Solids and Structures, 16(2), 119-130. 
Dixon, J. R., \& Pook, L. P. (1969). Stress intensity factors calculated generally by the finite element technique. Nature, 224(5215), 166-167.

Kotousov, A., Berto, F., Lazzarin, P., \& Pegorin, F. (2012). Three dimensional finite element mixed fracture mode under anti-plane loading of a crack. Theoretical and Applied Fracture Mechanics, 62, 26-33.

Kotousov, A., Lazzarin, P., Berto, F., \& Pook, L. P. (2013). Three-dimensional stress states at crack tip induced by shear and anti-plane loading. Engineering Fracture Mechanics, 108, 65-74.

Lazzarin, P., \& Zappalorto, M. (2012). A three-dimensional stress field solution for pointed and sharply radiused V-notches in plates of finite thickness. Fatigue \& Fracture of Engineering Materials \& Structures, 35(12), 1105-1119.

Paris, P.C.\& Sih, G.C. (1965) Stress analysis of cracks. In; Fracture toughness testing and its applications. ASTM STP 381. Philadelphia: American Society for Testing and Materials; p. 30-81.

Pook, L. P. (1994). Some implications of corner point singularities. Engineering Fracture Mechanics, 48(3), 367-378.

Pook, L. P. (1968). Brittle fracture of structural materials having a high strength weight ratio (Doctoral dissertation, $\mathrm{PhD}$ thesis, University of Strathclyde, Glasgow).

Pook, L.P. (2000) Linear elastic fracture mechanics for engineers. Theory and applications. Southampton: WIT Press.

Pook, L. P. (2010). Five decades of crack path research. Engineering Fracture Mechanics, 77(11), 1619-1630.

Pook, L. P. (2013). A 50-year retrospective review of three-dimensional effects at cracks and sharp notches. Fatigue \& Fracture of Engineering Materials \& Structures, 36(8), 699-723.

Pook, L. P., Berto, F., Campagnolo, A., \& Lazzarin, P. (2014). Coupled fracture mode of a cracked disc under anti-plane loading. Engineering Fracture Mechanics, 128, 22-36.

Pook, L. P. (2015). The linear elastic analysis of cracked bodies and crack paths. Theoretical and Applied Fracture Mechanics, 79, 34-50.

Pook, L. P., Campagnolo, A., Berto, F., \& Lazzarin, P. (2015). Coupled fracture mode of a cracked plate under anti-plane loading. Engineering Fracture Mechanics, 134, 391-403.

Pook, L. P., Campagnolo, A., \& Berto, F. (2016). Coupled fracture modes of discs and plates under anti-plane loading and a disc under in-plane shear loading. Fatigue \& Fracture of Engineering Materials \& Structures.

Pulver, S. (2008). Quaternions: The hypercomplex number system. The Mathematical Gazette, 92(525), 431-436.

Timoshenko S.P., \& Goodier J.N. (1970). Theory of elasticity. 4th ed. New York,: McGraw-Hill Book Company.

Weisz-Patrault, D., Bock, S., \& Gürlebeck, K. (2014). Three-dimensional elasticity based on quaternion-valued potentials. International Journal of Solids and Structures, 51(19), 3422-3430.

Williams, M.L. (1957). On the stress distribution at the base of a stationary crack. Journal of Applied Mechanics, 24, 109-114.

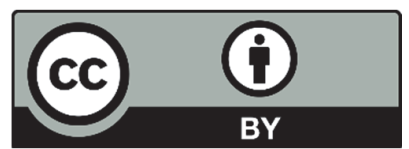

(C) 2017 by the authors; licensee Growing Science, Canada. This is an open access article distributed under the terms and conditions of the Creative Commons Attribution (CC-BY) license (http://creativecommons.org/licenses/by/4.0/). 